\title{
A NOVEL MATHEMATICAL APPROACH FOR FINITE ELEMENT FORMULATION OF FLEXIBLE ROBOT DYNAMICS
}

\author{
A. M. Chu ${ }^{\text {a, } 1}$, C. D. Nguyen $^{\text {a }}$, X. B. Duong ${ }^{b}$, A. V. Nguyen $^{c}$, T. A. \\ Nguyen $^{\mathrm{d}}$, Chi Hieu Le ${ }^{\mathrm{e}}$, Michael Packianather ${ }^{\mathrm{f}}$ \\ ${ }^{a}$ Institute of Simulation Technology, Le Quy Don Technical University, Ha Noi, Viet Nam; \\ ${ }^{b}$ Center of Advanced Technology, Le Quy Don Technical University, Hanoi, Vietnam \\ ${ }^{c}$ Department of Special Robotics \& Mechatronics, Le Quy Don Technical University, Hanoi, Vietnam. \\ ${ }^{d}$ Department of Physics, Le Quy Don Technical University, Ha Noi, Viet Nam \\ ${ }^{e}$ Faculty of Engineering and Science, University of Greenwich, Kent ME4 4TB, United Kingdom. \\ ${ }^{f}$ School of Engineering, Cardiff University, Cardiff CF24 3AA, United Kingdom
}

In conventional Finite Element - Lagrangian methods, the dynamics model of a flexible robot is usually formulated based on a critical assumption that the kinetic energy of an element is approximately calculated with an integral of mass point energy. Since the energy integral is implicit, the formulation of the dynamics model is also very complex and implicit. Hence, this paper develops a new mathematical approach for the dynamic modelling of a general flexible/rigid robot. The proposed method is more comprehensive and efficient in comparison with the previous ones because it no longer requires the calculation of the symbolic integrals and the implicit expressions of the elemental and global mass matrices. Besides, the proposed approach is applicable for both the flexible robots and the hybrid flexible/rigid robots. To validate the proposed method, numerical simulations and experimental results are presented.

Keywords: Flexible robots, flexible/rigid robots, kinematics of machines, dynamics of machines, finite elements method.

\section{Introduction}

In comparison to the conventional rigid robots or manipulators, the flexible ones have outstanding advantages such as a lower overall mass, smaller actuators, lower energy consumptions, and a greater payload-to-manipulator-weight ratio. However, the dynamic modelling of the flexible robots is much more complex.

The Finite Elements Method (FEM) has been widely used to model and analyse the dynamics of flexible robots [1-19]. As compared with the Assumed Modes Method [22-28], the main advantage of FEM is that it is ideally suited for the dynamic modelling of the flexible robots which consist of multi-links and different joint types $[1,18,19]$. In addition, FEM is 
capable of handling nonlinear conditions and irregularities in the structure and mixed boundary conditions.

In the literature, to establish equations of motion for the flexible robots, FEM has been used in conjunction with different analytical approaches, such as (i) the Lagrange's equations - based approach [1,7,12,13,15,22-28], (ii) the Newton-Euler's equations - based approach [3,6,17], and (iii) Kane's equations - based approach [16].

In addition, there have also been some different finite element approaches which were developed for the dynamic modelling of Flexible Multibody Systems, such as the Floating Frame of Reference (FFR) formulation [28-34], the Absolute Nodal Coordinate Formulation (ANCF) [35, 44-46], the Co-Rotational Finite Element Method (CRFEM) [37-42], and the Rigid Finite Element Method (RFEM) [36, 43]. A comprehensive review about these approaches was presented in [36]. In FFR formulations, two sets of coordinate systems are usually employed where one set characterizes the location and orientation of the bodies, while the other one describes the elastic deformation of the bodies [28-34]. In ANCF formulations [35,44-46], since the position of each point on a flexible body is calculated with a global shape function defined in a fixed reference frame, the global mass matrix of the dynamic equation is a constant matrix. In CRFEM method [37-42], a moving frame, the so called corotated frame, is attached to each element of a body in order to define the elastic deformation of the corresponding element. As for RFEM method [36, 43], each body is divided into rigid finite elements, and all the elements are connected with each other via springs and dampers.

Although many studies have been devoted to the dynamic modelling of flexible robots, few papers have carried on increasing the accuracy and reducing the computational complexity for the formulation of the dynamics model. Most of the previous FE formulations of the flexible robot dynamics are usually based on an important assumption that each link of a robot is assumed as an infinitely thin flexible rod. Further, to formulate elemental mass matrices for constructing the global mass matrix of the dynamic equation, a symbolic integration of the kinetic energy of a mass point along the length of an element is employed. In practice, this computational scheme has some considerable disadvantages. In particular, the construction of the global mass matrix of the dynamic equation from all the elemental mass matrices suffers from a computational burden since several complex and implicit mathematical transformations are required.

In this paper, a novel mathematical modelling method is developed and validated for application in formulating the dynamics model of the flexible/rigid robots. In the proposed approach, the motion of a flexible robot is decomposed into rigid and flexible motions. Both the flexible and rigid motions of each element of a link are taken into account when calculating the total kinetic energy of an element. By using the Jacobian matrices and a global vector of the generalized velocities, all elemental mass matrices and the global mass matrix for a general flexible/rigid robot is explicitly calculated to determine the mass and all inertia moments of all elements. In this manner, the dynamic equation for a rigid robot can be obtained by eliminating all the elastic displacements in the dynamic equation of a flexible robot. Thus, the proposed method is applicable for not only the flexible robots but also the flexible/rigid robots which consist of links of a given geometry. Furthermore, since all the elemental mass matrices can be formulated without any symbolic integrals, the time complexity of the formulation is reduced by $\mathrm{O}(\eta)$, where $\eta$ is the number of finite elements on all links. It was well demonstrated that the dynamic 
modelling scheme proposed in this study for a flexible robot is similar to the commonly used schemes of the dynamic modelling for the conventional rigid robots. Thus, the proposed method is very useful and helpful to users who have been familiar with the dynamic modelling and control of rigid robots. Therefore, the proposed method is more efficient, advantageous and useful when compared with previous methods. Finally, to present validations of the newly developed dynamic modelling method, the numerical examples and experimental results are presented.

\section{Equations of Motion}

This section presents a new mathematical approach to establish the dynamic equation of a flexible robot. In the proposed approach, the rigid and flexible motions of an element of a link are considered when calculating the linear velocity, the angular velocity, and the kinetic energy of each element of a link. Besides, the global mass matrix of the dynamic equation can be effectively calculated and explicitly expressed in terms of Jacobian matrices and the global generalized velocities without any complex assembling procedures.

Let's consider a planar flexible robot consisting of $n$ links and $n$ joints. Fig. 1 presents a generalized schematic of an arbitrary couple of flexible links of the robot, namely link $i-1$ and link $i$. In this figure link $i-1$ connects with link $i$ by a joint $i$ which can be a revolute joint, a sliding prismatic joint or a fixed prismatic joint [1]. Link $i$ of length $l_{i}$ is divided into $n_{i}$ elements of equal length $l_{i e}$. The vector of flexural displacements and the flexural slopes of an element $j$ is $\mathbf{p}_{i j}=\left[\begin{array}{llll}u_{(i) 2 j-1} & u_{(i) 2 j} & u_{(i) 2 j+1} & u_{(i) 2 j+2}\end{array}\right]^{T}$.

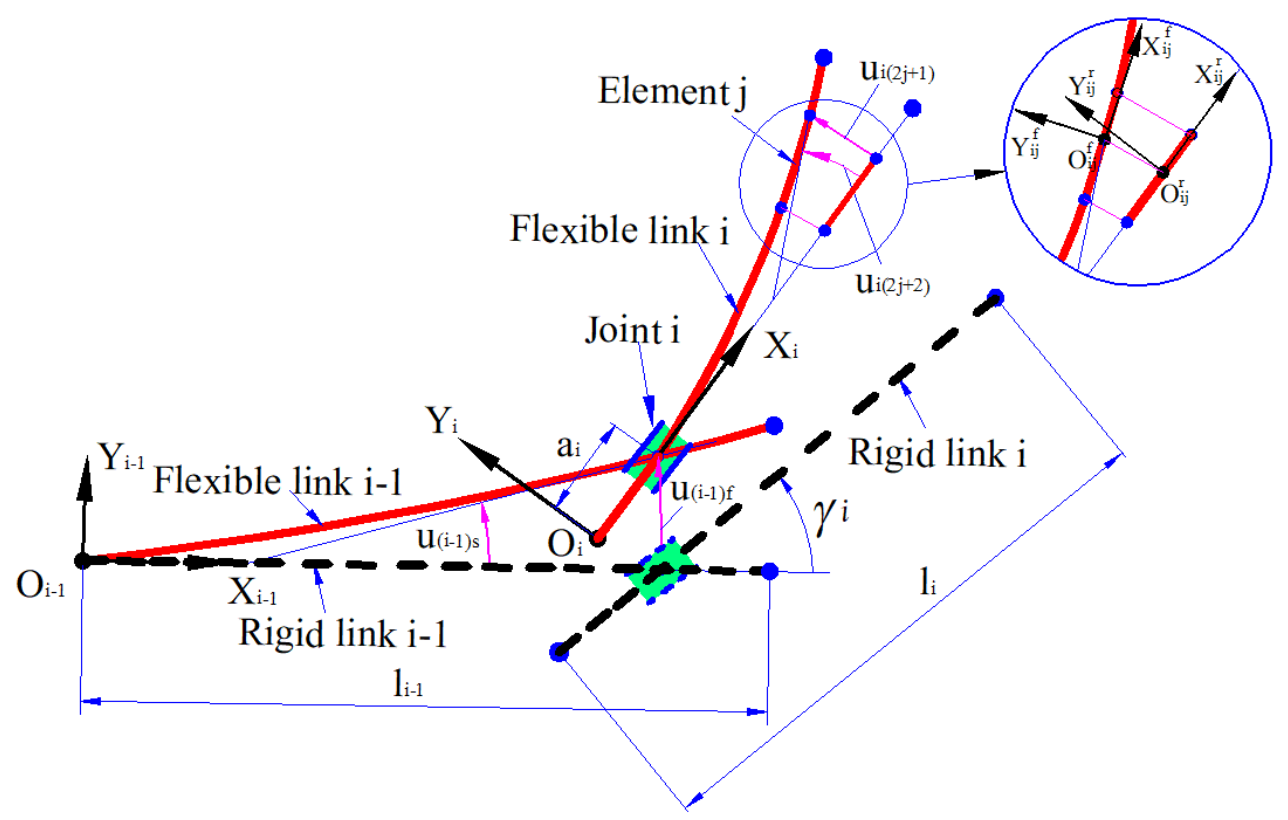

Figure. 1. Link $i-1$ and link $i$ of a flexible robot

Suppose that the homogeneous motion of an element $j$ includes the two motions. The first motion is a rigid motion which implies that the motion of the element $j$ will take place regardless of the elastic deformation of the link $i$. The second motion is a 
flexible motion relative to the rigid motion, which is caused by the elasticity effect of the link $i$.

Let's define $O_{i} X_{i} Y_{i} Z_{i}$ as the local coordinate system attached to the link $i$, where the origin $O_{i}$ is fixed to the proximal end of the link $i$ and the axis $X_{i}$ points in the direction of the link $i$. Similarly, $O_{i-1} X_{i-1} Y_{i-1} Z_{i-1}$ is defined for the link $i-1 . O_{0} X_{0} Y_{0} Z_{0}$ is the reference coordinate system fixed to the base.

Let $O_{i j}^{r} x_{i j}^{r} y_{i j}^{r} z_{i j}^{r}$ be a local frame, of which the origin point $O_{i j}^{r}$ is located at the centre of mass of the rigid element $j$, and the axis $x_{i j}^{r}$ points in the direction of the rigid link $i$. Similarly, the frame $O_{i j}^{f} x_{i j}^{f} y_{i j}^{f} z_{i j}^{f}$ is defined for the flexible element $j$. The superscript " $\mathrm{r}$ " stands for a rigid element, and " $\mathrm{f}$ " stands for a corresponding flexible element.

In essence, the frame $O_{i j}^{f} x_{i j}^{f} y_{i j}^{f} z_{i j}^{f}$ can be obtained by translating and rotating the frame $O_{i j}^{r} x_{i j}^{r} y_{i j}^{r} z_{i j}^{r}$ at a flexural displacement $w_{i j}(x)$ along $y_{i j}^{r}$ axis, and at a flexural slope $w_{i j}^{\prime}(x)$ around $z_{i j}^{r}$ axis, respectively. The transformation matrix can be expressed as follows:

$$
\mathbf{H}_{i j}^{f}=\left[\begin{array}{cccc}
\cos w_{i j}^{\prime} & -\sin w_{i j}^{\prime} & 0 & 0 \\
\sin w_{i j}^{\prime} & \cos w_{i j}^{\prime} & 0 & w_{i j} \\
0 & 0 & 1 & 0 \\
0 & 0 & 0 & 1
\end{array}\right]=\left[\begin{array}{cc}
\mathbf{A}_{i j}^{f} & \mathbf{u}_{i j}^{f} \\
0 & 1
\end{array}\right]
$$

where the flexural displacement $w_{i j}(x)$ is calculated at the centre of mass , $x=l_{i e} / 2$, as $w_{i j}(x)=\sum_{m=1}^{4} \phi_{m}(x) u_{(i)(2 j-2+m)}$, where $\phi_{m}$ are the shape functions.

In the frame $O_{i} X_{i} Y_{i} Z_{i}$ attached to a link $i$, a point $\mathbf{r}_{i j}(x)$ on an element $j$ of a link $i$ is calculated as follows:

$$
\mathbf{r}_{(i) i j}(x)=\left[\begin{array}{c}
(j-1) l_{i e}+x \\
w_{i j}(x) \\
0 \\
1
\end{array}\right]
$$

In the precede frame $O_{i-1} X_{i-1} Y_{i-1} Z_{i-1}$, the point $\mathbf{r}_{(i) i j}(x)$ is denoted as $\mathbf{r}_{(i-1) i j}(x)$ which can be determined with the following kinematic relationship:

$$
\mathbf{r}_{(i-1) i j}(x)=\boldsymbol{\Theta}_{(i-1) i} \mathbf{r}_{(i) i j}(x),
$$


where $\boldsymbol{\Theta}_{(i-1) i}$ is the transformation matrix describing the kinematic relationship between a link $i-1$ and a link $i$ for a flexible robot, which was proven in our previous study [1].

In order to determine the position and the orientation of a flexible element $j$, the position and the orientation of the rigid element $j$ should be calculated first. In $O_{0} X_{0} Y_{0} Z_{0}$ , the point $\mathbf{r}_{0 i j}^{r} \equiv O_{i j}^{r}$, and the orientation of $O_{i j}^{r} x_{i j}^{r} y_{i j}^{r} z_{i j}^{r}$ is characterized by the following transformation matrix:

$$
\mathbf{H}_{0 i j}^{r}=\left(\prod_{\xi=1}^{i} \Theta_{(\xi-1) \xi}\right) \mathbf{H}_{i j}^{r}=\left[\begin{array}{cc}
\mathbf{A}_{0 i j} & \mathbf{r}_{0 i j}^{r} \\
0 & 1
\end{array}\right],
$$

where

$$
\mathbf{H}_{i j}^{r}=\left[\begin{array}{cccc}
1 & 0 & 0 & (j-1) l_{i e}+x \\
0 & 1 & 0 & 0 \\
0 & 0 & 1 & 0 \\
0 & 0 & 0 & 1
\end{array}\right] .
$$

Consequently, in $O_{0} X_{0} Y_{0} Z_{0}$, the position vector $\mathbf{r}_{0 i j}^{f}$ (the point $O_{i j}^{f}$ ) can be determined as follows:

$$
\mathbf{r}_{0 i j}^{f}=\mathbf{r}_{0 i j}^{r}+\mathbf{A}_{0 i j} \mathbf{u}_{i j}^{f}
$$

It is clearly seen that, the centre of mass of any element of a given link can be determined explicitly with Eq. (6).

Let's consider the dynamic equation of the flexible robot:

$$
\mathbf{M}(\mathbf{q}) \ddot{\mathbf{q}}+\mathbf{C}(\mathbf{q}, \dot{\mathbf{q}}) \dot{\mathbf{q}}+\mathbf{K q}+\mathbf{G}(\mathbf{q})=\mathbf{F}
$$

Eq. (7) is written with respect to the global vector of the generalized coordinates, $\mathbf{q}=\left[\begin{array}{lllllll}p_{1} & \kappa_{1}^{T} & p_{2} & \kappa_{2}^{T} & \cdots & p_{n} & \kappa_{n}^{T}\end{array}\right]^{T}$, where $p_{i}$ is the joint variable $i$, and $\kappa_{i}=\left[\begin{array}{lllll}u_{(i) 1} & u_{(i) 2} & \ldots & u_{(i)\left(2 n_{i}+1\right)} & u_{(i)\left(2 n_{i}+2\right)}\end{array}\right]^{T}$ is the vector of the elastic displacements of all elements of a link $i$. The applied forces/torques imposing on $n$ joints of the robot are

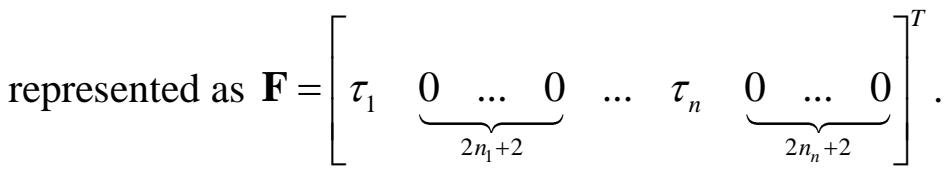

\section{Formulation of the global mass matrix $M(q)$}

Taking time derivative both sides of Eq. (6) yields:

$$
\dot{\mathbf{r}}_{0 i j}^{f}=\dot{\mathbf{r}}_{0 i j}^{r}+\dot{\mathbf{A}}_{0 i j} \mathbf{u}_{i j}^{f}+\mathbf{A}_{0 i j} \dot{\mathbf{u}}_{i j}^{f},
$$

Note that $\mathbf{u}_{i j}^{f}=\mathbf{A}_{0 i j}^{T} \mathbf{u}_{0 i j}^{f}$. Therefore, 


$$
\begin{gathered}
\mathbf{v}_{0 i j}^{f}=\mathbf{v}_{0 i j}^{r}+\dot{\mathbf{A}}_{0 i j} \mathbf{A}_{0 i j}^{T} \mathbf{u}_{0 i j}^{f}+\mathbf{A}_{0 i j} \dot{\mathbf{u}}_{i j}^{f} \\
\mathbf{v}_{0 i j}^{f}=\mathbf{v}_{0 i j}^{r}+\tilde{\boldsymbol{\omega}}_{0 i j}^{r} \mathbf{u}_{0 i j}^{f}+\mathbf{A}_{0 i j} \dot{\mathbf{u}}_{i j}^{f} \\
\mathbf{v}_{0 i j}^{f}=\mathbf{v}_{0 i j}^{r}-\tilde{\mathbf{u}}_{0 i j}^{f} \boldsymbol{\omega}_{0 i j}^{r}+\mathbf{A}_{0 i j} \dot{\mathbf{u}}_{i j}^{f}
\end{gathered}
$$

where $\mathbf{v}_{0 i j}^{f}$ is the velocity of the centre of mass $O_{i j}^{f}$ of an element $j . \boldsymbol{\omega}_{0 i j}^{r}$ is the angular velocity of an element $j$ with regardless of the elastic deformation of link $i$.

It is noticeable that

$$
\mathbf{v}_{0 i j}^{r}=\frac{\partial \mathbf{r}_{0 i j}^{r}}{\partial \mathbf{q}} \dot{\mathbf{q}}, \boldsymbol{\omega}_{0 i j}^{r}=\frac{\partial \boldsymbol{\omega}_{0 i j}^{r}}{\partial \dot{\mathbf{q}}} \dot{\mathbf{q}}, \text { and } \dot{\mathbf{u}}_{i j}^{f}=\frac{\partial \mathbf{u}_{i j}^{f}}{\partial \mathbf{q}} \dot{\mathbf{q}} \text {. }
$$

Hence, the velocity $\mathbf{v}_{0 i j}^{f}$ can be expressed in terms of Jacobian matrices and the generalized velocities as follows:

$$
\mathbf{v}_{0 i j}^{f}=\left(\mathbf{J}_{i j(T)}^{r}-\tilde{\mathbf{u}}_{0 i j}^{f} \mathbf{J}_{i j(R)}^{r}+\mathbf{A}_{0 i j} \mathbf{J}_{i j}^{f}\right) \dot{\mathbf{q}}
$$

where the Jacobian matrices are determined as follows:

$$
\mathbf{J}_{i j(T)}^{r}=\frac{\partial \mathbf{r}_{0 i j}^{r}}{\partial \mathbf{q}}, \mathbf{J}_{i j(R)}^{r}=\frac{\partial \boldsymbol{\omega}_{0 i j}^{r}}{\partial \dot{\mathbf{q}}} \text { and } \mathbf{J}_{i j}^{f}=\frac{\partial \mathbf{u}_{i j}^{f}}{\partial \mathbf{q}} .
$$

The matrices $\mathbf{J}_{i j(T)}^{r}$ and $\mathbf{J}_{i j(R)}^{r}$ are the translational and rotational Jacobian matrices of an element $j$ regardless of the elastic displacements of the element $j$. Meanwhile the Jacobian matrix $\mathbf{J}_{i j}^{f}$ is calculated with respect to the relative flexural displacement of the element $j$.

Let's denote

$$
\mathbf{J}_{i j(T)}^{f}=-\tilde{\mathbf{u}}_{0 i j}^{f} \mathbf{J}_{i j(R)}^{r}+\mathbf{A}_{0 i j} \mathbf{J}_{i j}^{f}
$$

$\mathbf{J}_{i j(T)}^{f}$ plays a role of a translational Jacobian matrix with regards to to the elastic deformation of the element $j$ of the link $i$. The absolute linear velocity $\mathbf{v}_{0 i j}^{f}$ can be rewritten as follows:

$$
\mathbf{v}_{0 i j}^{f}=\left(\mathbf{J}_{i j(T)}^{r}+\mathbf{J}_{i j(T)}^{f}\right) \dot{\mathbf{q}}
$$

The absolute angular velocity $\boldsymbol{\omega}_{0 i j}^{f}$ of a flexible element $j$ of a flexible link $i$ can be determined as follows:

$$
\boldsymbol{\omega}_{0 i j}^{f}=\boldsymbol{\omega}_{0 i j}^{r}+\boldsymbol{\omega}_{i j}^{f},
$$

where

$$
\tilde{\boldsymbol{\omega}}_{i j}^{f}=\dot{\mathbf{A}}_{i j}^{f}\left(\mathbf{A}_{i j}^{f}\right)^{T}
$$


In terms of the Jacobian matrices and the generalized velocities $\dot{\mathbf{q}}$, the angular velocity $\boldsymbol{\omega}_{0 i j}^{f}$ can be rewritten as follows:

$$
\boldsymbol{\omega}_{0 i j}^{f}=\left(\mathbf{J}_{i j(R)}^{r}+\mathbf{J}_{i j(R)}^{f}\right) \dot{\mathbf{q}},
$$

Where

$$
\mathbf{J}_{i j(R)}^{f}=\frac{\partial \boldsymbol{\omega}_{i j}^{f}}{\partial \dot{\mathbf{q}}}
$$

Assuming that the size of an element $j$ is small, and link $i$ is dived into a finite number of elements, the kinetic energy of an element $j$ of flexible link $i$ can be approximated as follows:

$$
T_{i j} \square \frac{1}{2} m_{i} l_{i e}\left(\mathbf{v}_{0 i j}^{f}\right)^{T} \mathbf{v}_{0 i j}^{f}+\frac{1}{2}\left(\boldsymbol{\omega}_{0 i j}^{f}\right)^{T} \mathbf{I}_{i j} \boldsymbol{\omega}_{0 i j}^{f}
$$

Substituting Eqs. (14 and17) into Eq. (19) yields

$$
T_{i j}=\frac{1}{2} m_{i} l_{i e}\left[\left(\mathbf{J}_{i j(T)}^{r}+\mathbf{J}_{i j(T)}^{f}\right) \dot{\mathbf{q}}\right]^{T}\left[\left(\mathbf{J}_{i j(T)}^{r}+\mathbf{J}_{i j(T)}^{f}\right) \dot{\mathbf{q}}\right]+\frac{1}{2}\left[\left(\mathbf{J}_{i j(R)}^{r}+\mathbf{J}_{i j(R)}^{f}\right) \dot{\mathbf{q}}\right]^{T} \mathbf{I}_{i j}\left[\left(\mathbf{J}_{i j(R)}^{r}+\mathbf{J}_{i j(R)}^{f}\right) \dot{\mathbf{q}}\right]
$$

Let's denote

$$
\begin{gathered}
\mathbf{J}_{i j(T)}=\mathbf{J}_{i j(T)}^{r}+\mathbf{J}_{i j(T)}^{f} \text {, and } \\
\mathbf{J}_{i j(R)}=\mathbf{J}_{i j(R)}^{r}+\mathbf{J}_{i j(R)}^{f}
\end{gathered}
$$

Rewriting $T_{i j}$ in terms of the elemental mass matrix gives

$$
T_{i j}=\frac{1}{2} \dot{\mathbf{q}}^{T} \mathbf{M}_{i j}(\mathbf{q}) \dot{\mathbf{q}}
$$

Therefore, the elemental mass matrix $\mathbf{M}_{i j}$ can be expressed as follows:

$$
\mathbf{M}_{i j}=m_{i} l_{i e} \mathbf{J}_{i j(T)}^{T} \mathbf{J}_{i j(T)}+\mathbf{J}_{i j(R)}^{T} \mathbf{I}_{i j} \mathbf{J}_{i j(R)}
$$

The total kinetic energy of $n$ links of the robot is calculated as follows:

$$
T=\sum_{i=1}^{n} \sum_{j=1}^{n_{j}} T_{i j}=\frac{1}{2} \dot{\mathbf{q}}^{T} \mathbf{M}(\mathbf{q}) \dot{\mathbf{q}}
$$

Finally, the global mass matrix is obtained as follows:

$$
\begin{aligned}
\mathbf{M}(\mathbf{q}) & =\sum_{i=1}^{n} \sum_{j=1}^{n_{j}} \mathbf{M}_{i j}(\mathbf{q}) \\
& =\sum_{i=1}^{n} \sum_{j=1}^{n_{i}} m_{i} l_{i e} \mathbf{J}_{i j(T)}^{T} \mathbf{J}_{i j(T)}+\mathbf{J}_{i j(R)}^{T} \mathbf{I}_{i j} \mathbf{J}_{i j(R)}
\end{aligned}
$$

Remark. It is clearly seen that the form of the global mass matrix of the dynamic equation of a flexible robot is similar to the form of the global mass matrix of the dynamic equation of a rigid robot. The dynamic equation for the flexible robots is more generalized 
than the dynamic equation for the rigid robots. The dynamic equation of a rigid robot can be obtained by eliminating all the components $\mathbf{J}_{i j(T)}^{f}$ and $\mathbf{J}_{i j(R)}^{f}$ in Eqs. (20 and 21) that characterize the elasticity effects in the dynamic equation of a flexible robot. Therefore, Eq. (25) is a generic formulation of the global mass matrix of the dynamic equation which can be used for both the flexible robot and the rigid robot. Furthermore, this formulation is also applicable for the case when a part of some rigid links of a robot is flexible.

A flowchart of the algorithm for the symbolic formulation of the global mass $\mathbf{M}(\mathbf{q})$ is presented in Fig. 2.

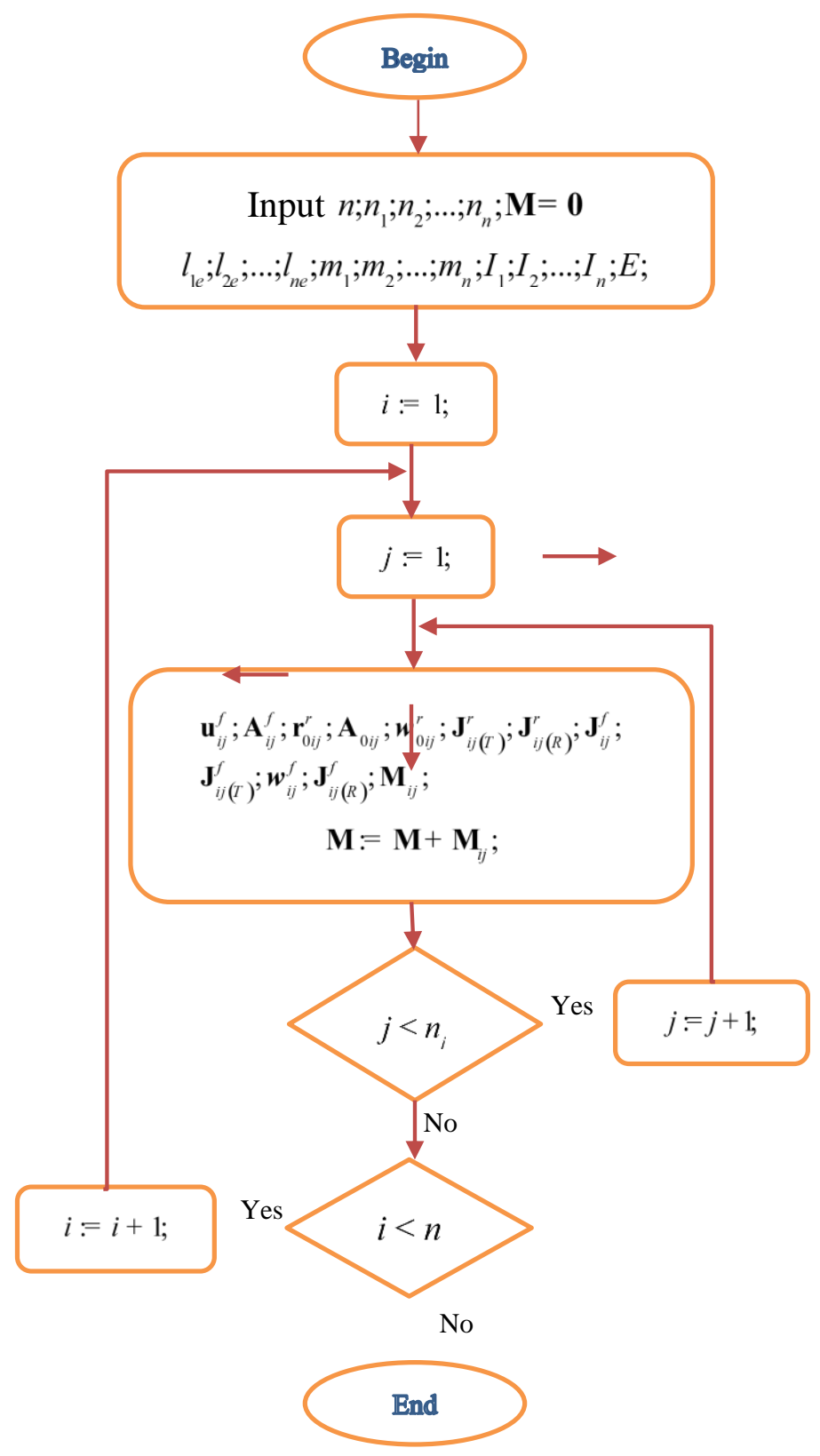

Figure 2. The algorithm for symbolic formulation of the global mass matrix 
For every element $j$ of link $i$, the vector $\mathbf{u}_{i j}^{f}$ and the matrix $\mathbf{A}_{i j}^{f}$ are calculated with Eq. (1), the position vector $\mathbf{r}_{0 i j}^{r}(x)$ and the rotation matrix $\mathbf{A}_{0 i j}$ are calculated with Eq. (4). The angular velocity $\boldsymbol{\omega}_{0 i j}^{r}$ is then determined via $\tilde{\boldsymbol{\omega}}_{0 i j}^{r}=\dot{\mathbf{A}}_{0 i j} \mathbf{A}_{0 i j}^{T}$. The Jacobian matrices $\mathbf{J}_{i j(T)}^{r}, \mathbf{J}_{i j(R)}^{r}$ and $\mathbf{J}_{i j}^{f}$ are calculated with Eq. (11), and the matrix $\mathbf{J}_{i j(T)}^{f}$ is calculated with Eq. (12). The relative angular velocity $\boldsymbol{\omega}_{i j}^{f}$ is calculated with Eq. (15) and the Jacobian $\mathbf{J}_{i j(R)}^{f}$ is determined with Eq. (17). The elemental mass matrix $\mathbf{M}_{i j}$ is calculated with Eq. (23). For all elements on all links, the above steps are recursively looped to archive all $\mathbf{M}_{i j}$. Finally, the global matrix $\mathbf{M}$ is obtained with Eq. (25).

Note that, to complete the formulation of the dynamic equation Eq. (7), the global stiffness matrix $\mathbf{K}$ and the Coriolis and centrifugal matrix $\mathbf{C}$ are formulated by using the method presented in our previous work [1].

It is also noticeable that the dynamic modelling method of flexible robots proposed in this section is different from the Floating Frame of Reference (FFR) formulations [28-34]. In FFR formulations, two sets of coordinates are usually used to describe the configuration of the deformable bodies [28]; the first set describes the position of the bodies, while the second set describes the deformation of the bodies with respect to their coordinate systems. As discussed in [28], one critical issue that arises when using FFR formulations is the definition of the sets of the coordinate systems on the bodies, since the shape of deformation of a body is defined in its coordinate system. Therefore, in the dynamic modelling approach proposed in this paper, the following differences are made, in comparison with the FFR formulations [28-34]:

(i) One more set of element coordinate systems $O_{i j}^{r} x_{i j}^{r} y_{i j}^{r} z_{i j}^{r}$ is defined. Moreover, all the link frames and element frames are defined in a way that the novel transformation matrix that was proven in our previous work [1] can be used effectively to simplify the kinematic formulation of a multilink flexible robot with different joint types.

(ii) Jacobian matrices are taken into account for the dynamic modelling of a flexible robot. In this manner, the form of the dynamic equation of a flexible robot is similar to the form of the dynamic equation of a rigid robot.

\section{Numerical examples}

In this section, to validate the dynamic modelling method proposed in this study, the behaviour of the forward dynamics of two different flexible robots are analysed and simulated. The first robot has one flexible link and the second robot has two flexible links. In the examples, the numerical solution of the dynamic equation derived by using the proposed method is compared with the solutions of the dynamic equations established by using the previous method $[1,7-9,15]$.

\subsection{Example 1: Dynamics of a robot with one flexible link}

In this example, as shown in Fig. 3, the robot has two links, but the first link is rigid. Only the second link is flexible. The parameters of the robot are as follows. Link 1 has a length 
of $0.2 \mathrm{~m}$ and a mass of $2.0 \mathrm{~kg}$. The length of link 2 is $1.0 \mathrm{~m}$. The mass per meter of link 2 is $0.706 \mathrm{~kg} / \mathrm{m}$. Link 2 has a cross section area which is $9 \times 10^{-5} \mathrm{~m}^{2}$, and it is divided into 5 elements.

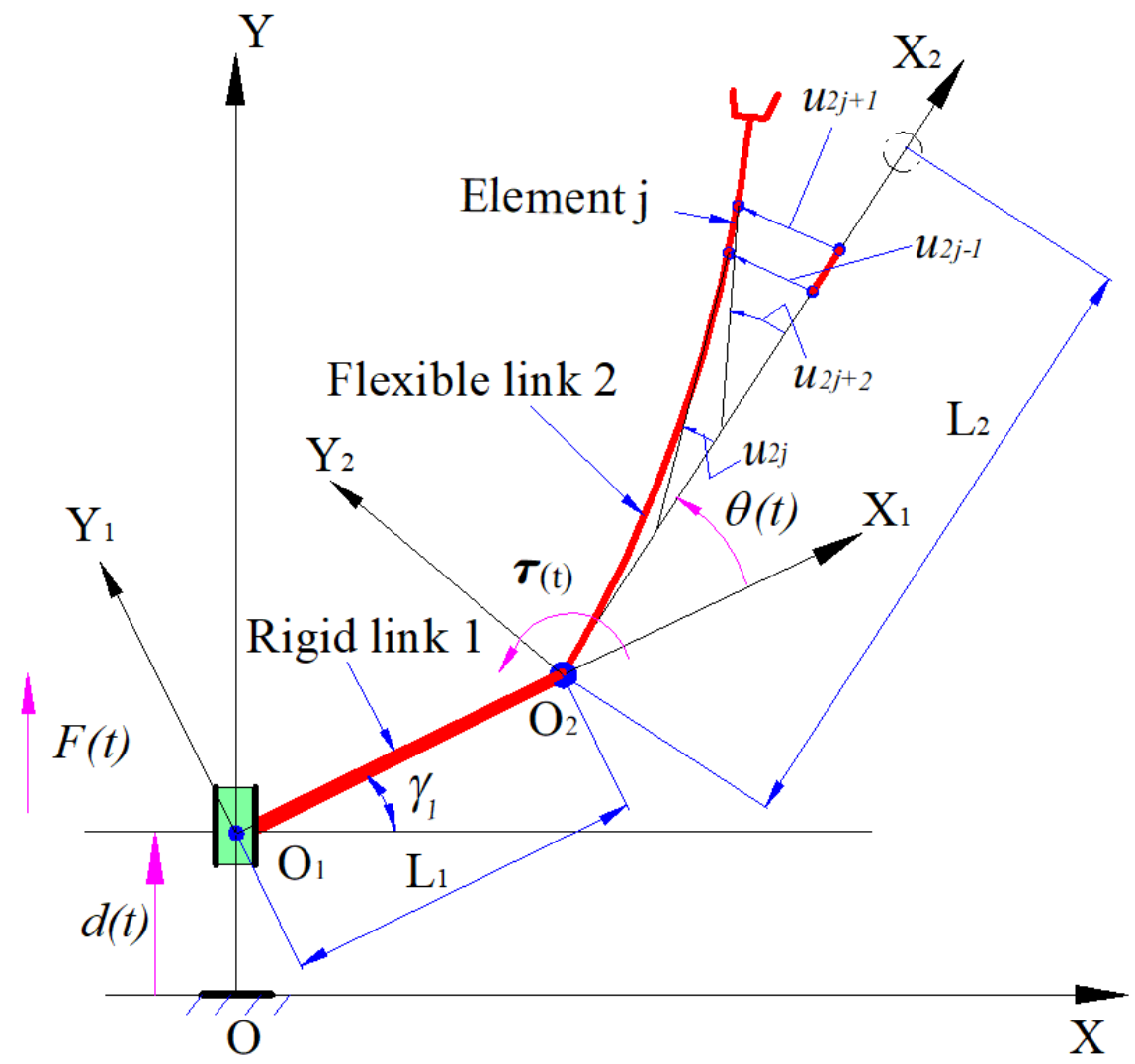

Figure 3. A flexible robot for numerical simulation

When the force/torque (Bang-bang rule) was applied as shown in Fig. 4 and Fig.5 for the inputs of the simulation, the two joints of the robot were displaced as shown in Figs. 6 and 7. Fig. 8 shows the flexural displacement at the end-effector of the robot. These graphs show that the time evolution of the displacement curves computed by using the method proposed in this study having a close resemblance to the curves computed by using the previous methods. Note that the simulation curves of the two numerical solutions continue to match well even when the parameters of the robot are changed.

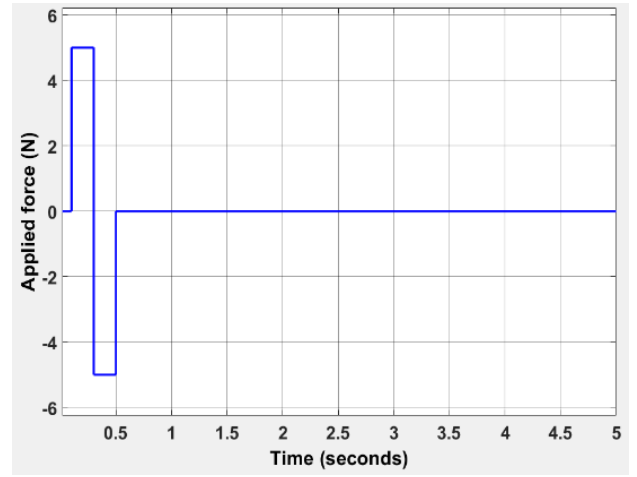

Figure 4. The input of applied force on the prismatic joint

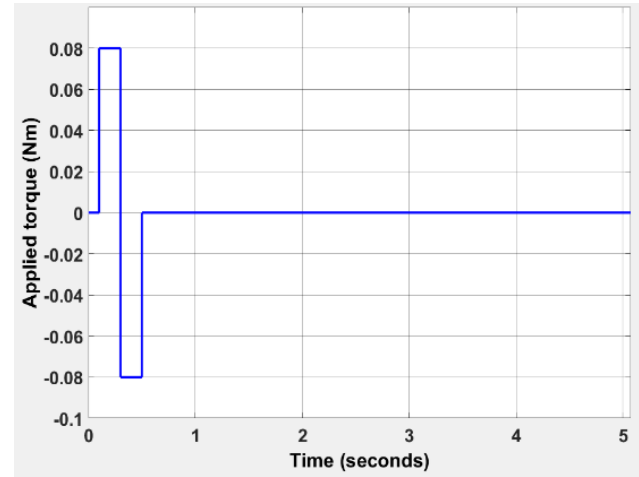

Figure 5. The input of applied torque on the revolution joint 


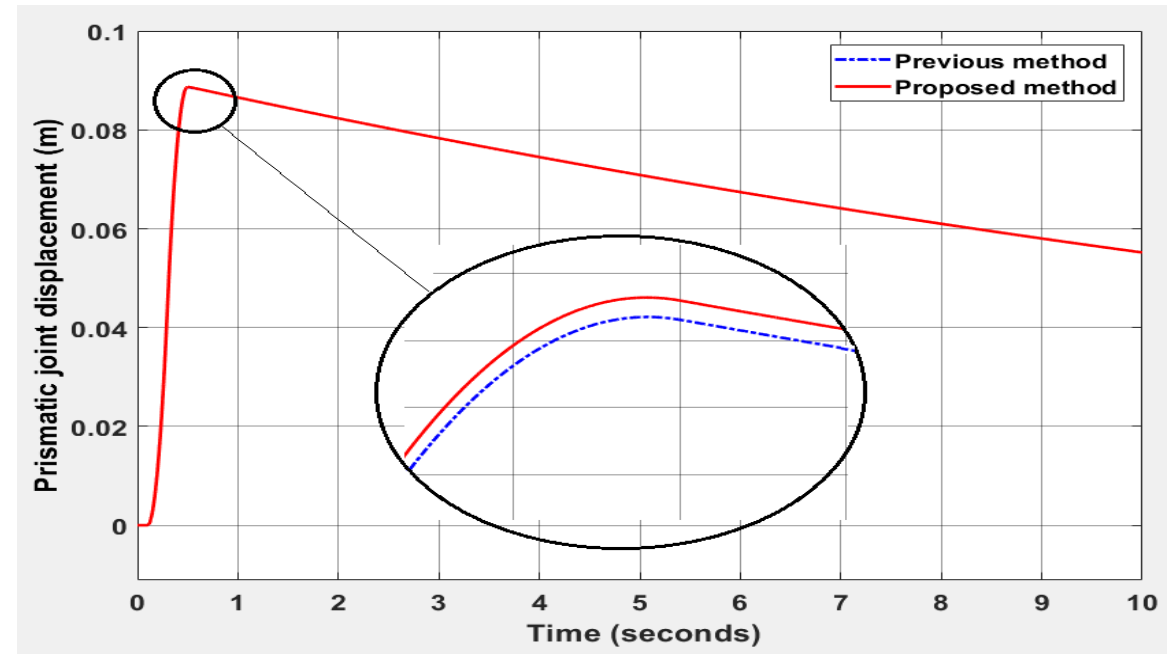

Figure 6. The displacement of the prismatic joint

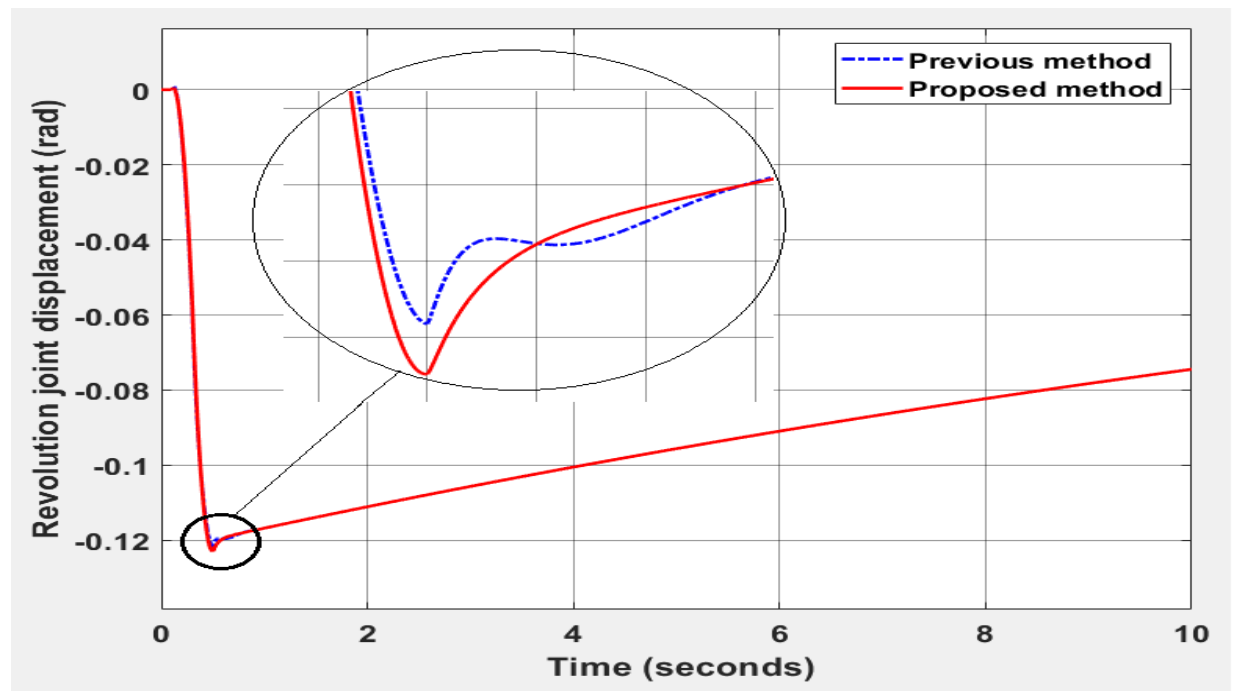

Figure 7. The displacement of the revolute joint

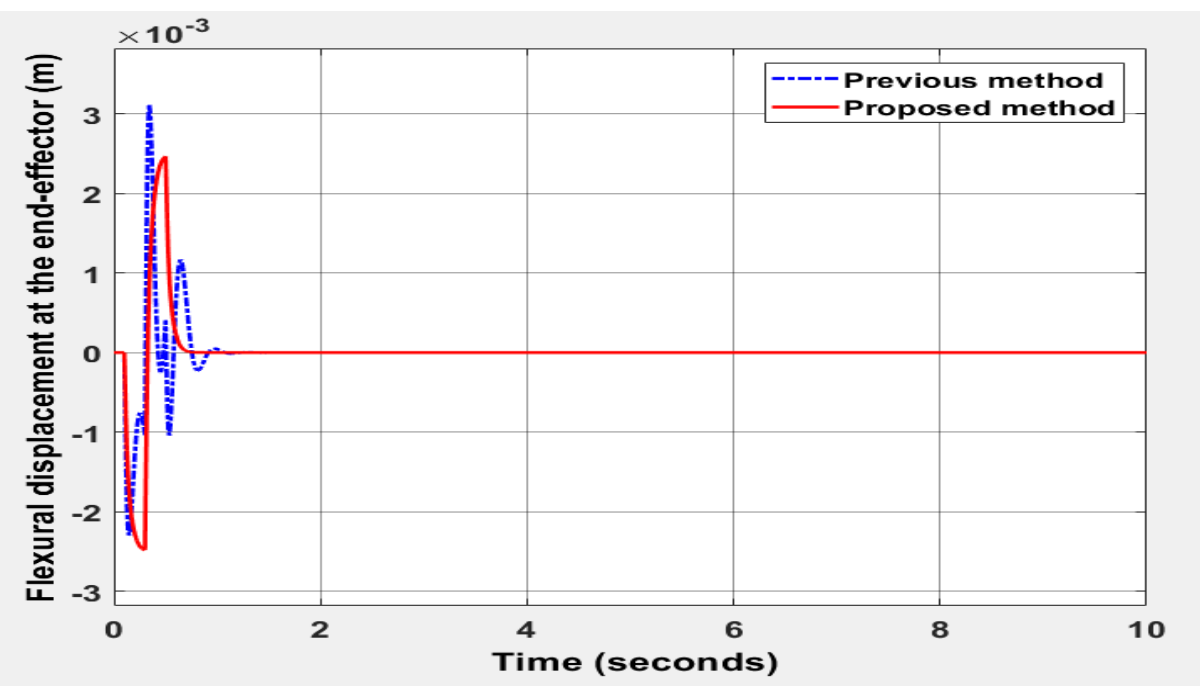

Figure 8. The flexural displacement at the end-effector 


\subsection{Example 2: Dynamics of a robot with two flexible links}

In order to demonstrate the proposed dynamic formulation with a more complex flexible robot, a two-link flexible robot with two rotational joints as shown in Fig. 9 is analysed. The lengths of flexible link 1 and flexible link 2 are $0.8 \mathrm{~m}$ and $0.3 \mathrm{~m}$, respectively. The cross-section areas of the two links are equal as $3 \times 10^{-5} \mathrm{~m}^{2}$. Each flexible link is divided into 2 elements. The mass of the payload at the end-effector is $50 \mathrm{~g}$. The applied torques at joint 1 and joint 2 are shown in Fig. 10 and Fig. 11, respectively. The displacements of the joints are displayed in Fig. 12 and Fig. 13. The flexural and slope displacement at the end-effector are presented in Fig. 14 and Fig. 15.

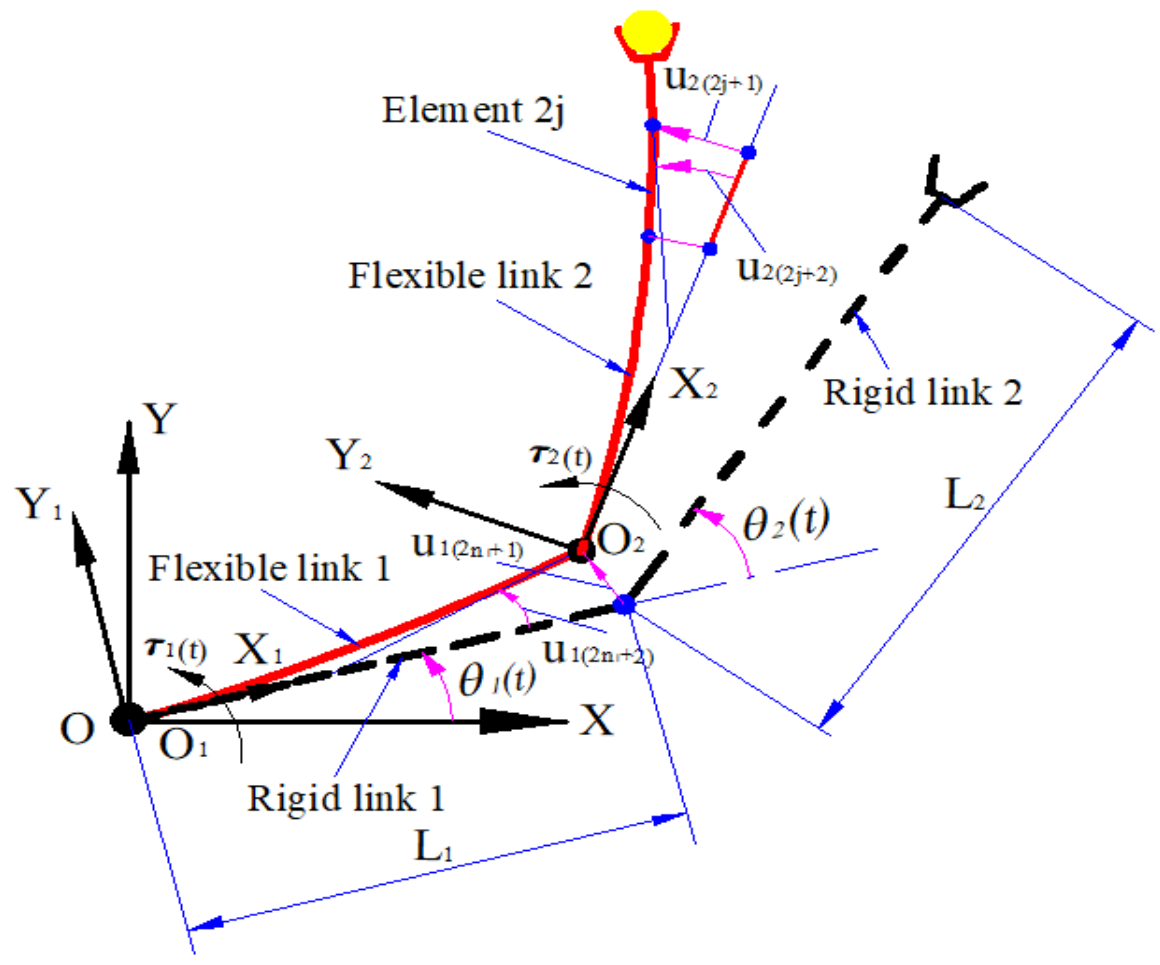

Figure 9. A two-link flexible robot with two rotational joints

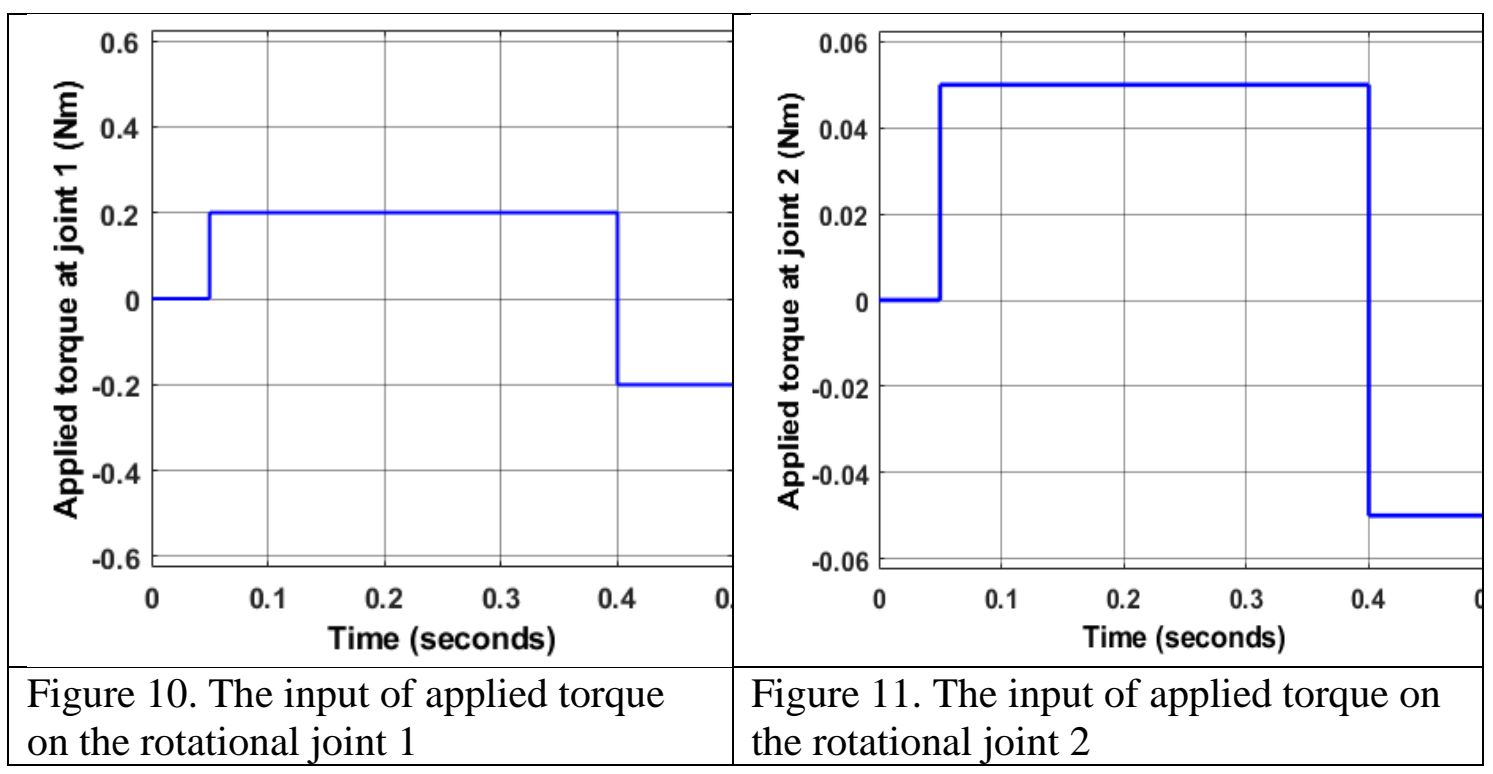




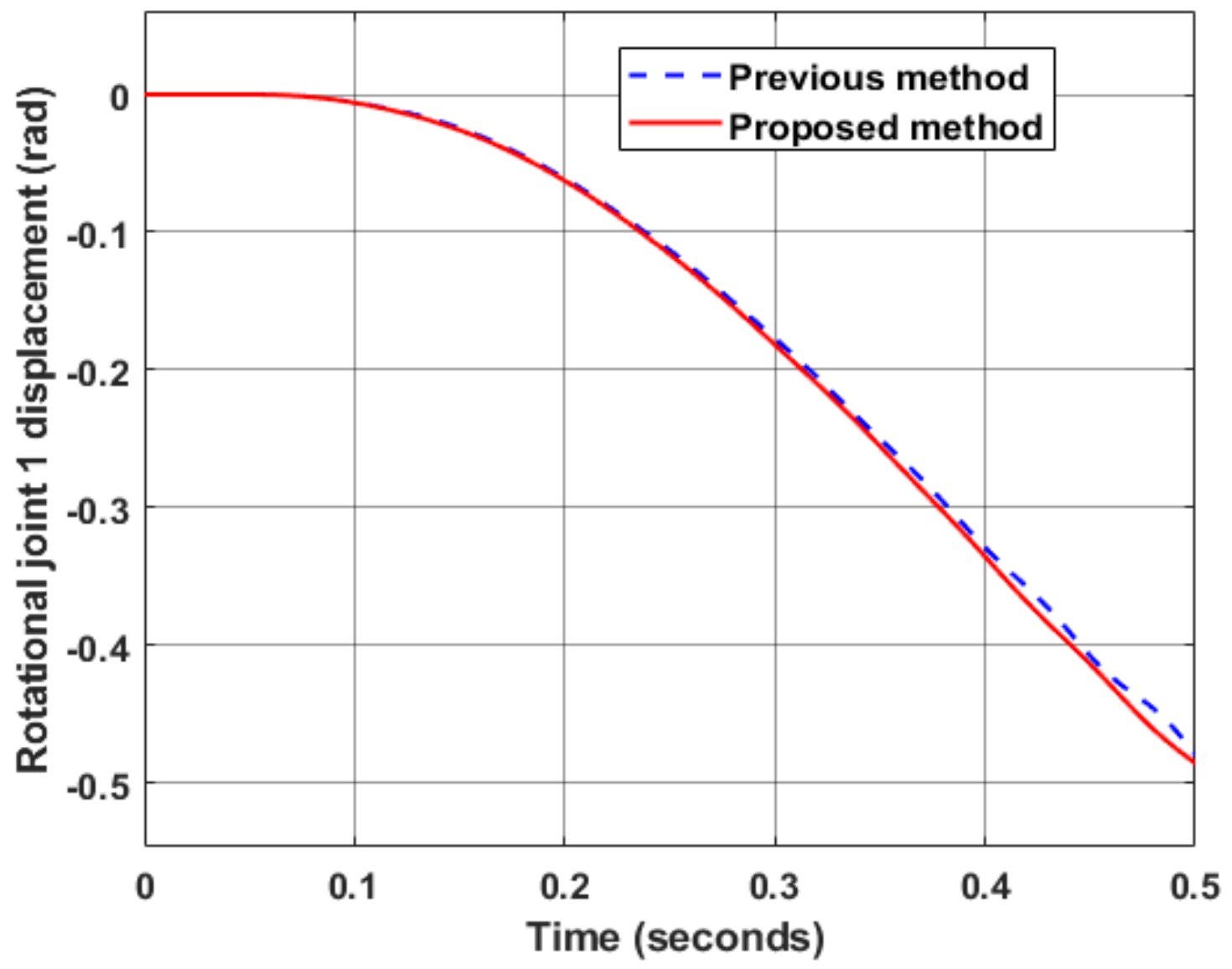

Figure 12. The displacement of the rotational joint 1

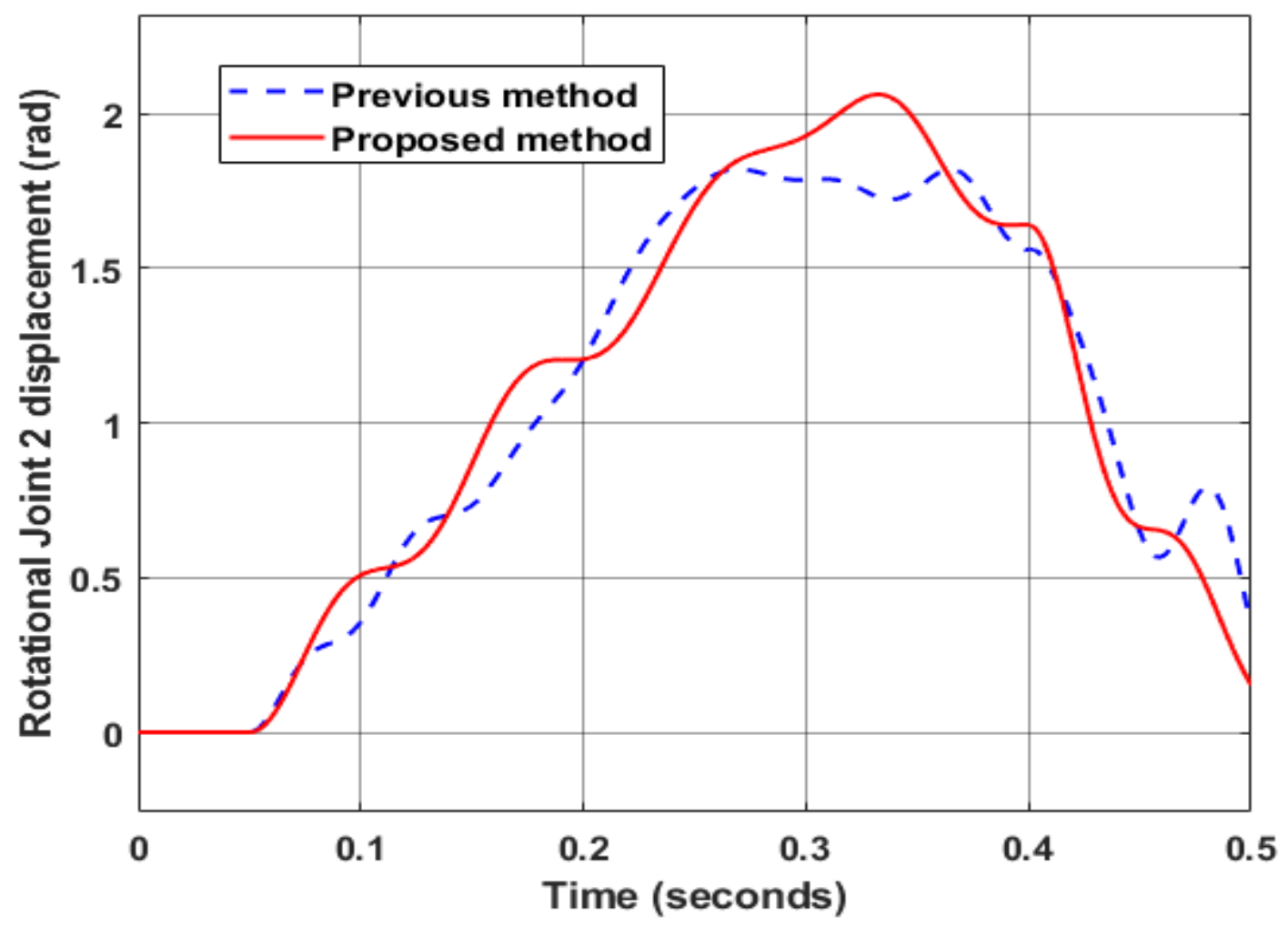

Figure 13. The displacement of the rotational joint 2 


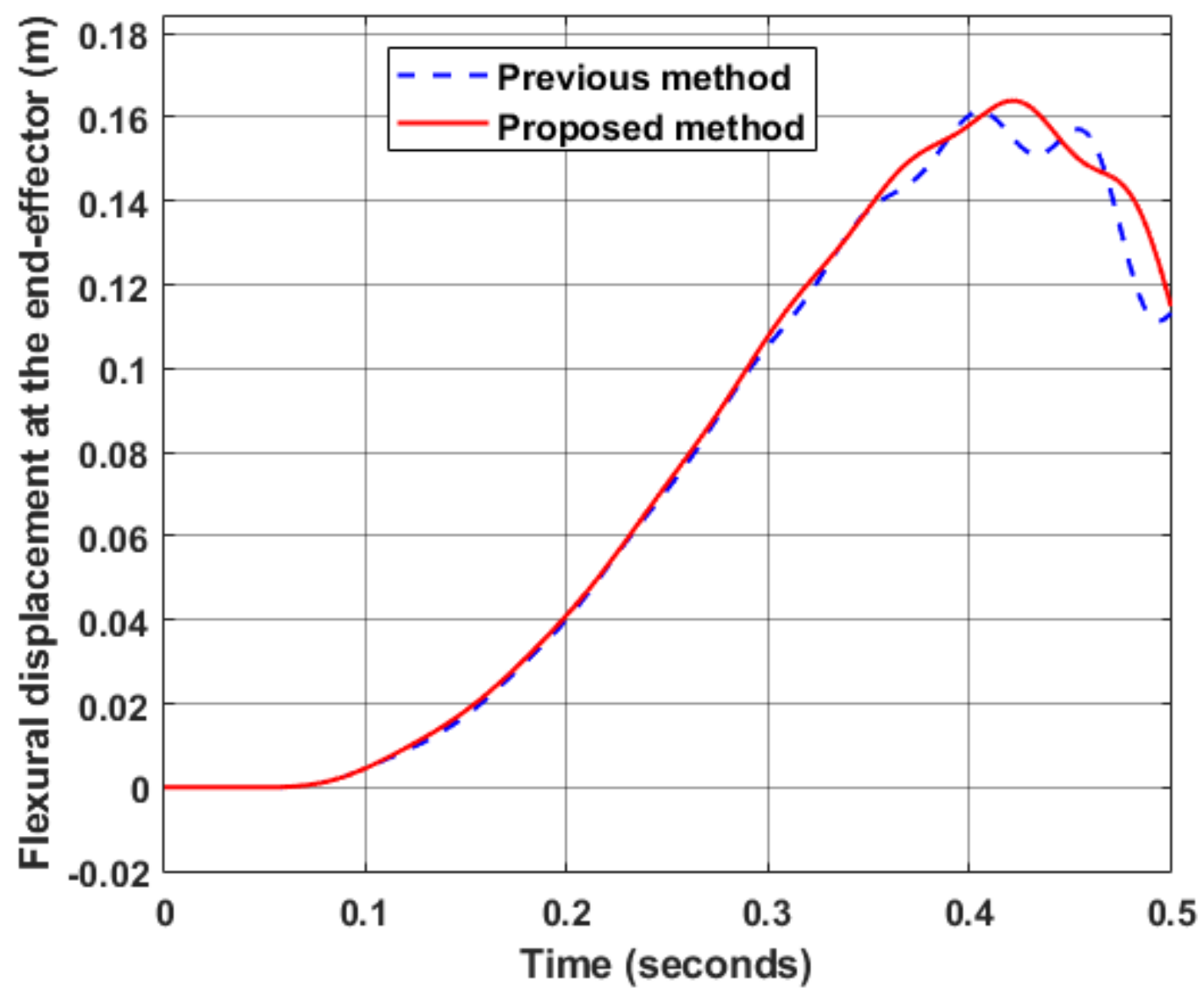

Figure 14. The flexural displacement at the end-effector

These figures show that, with a more complex flexible robot, the shape and the value of the displacement curves computed by using the method proposed in this study having a close resemblance to the curves computed by using the previous methods.

\section{Experimental Results}

In the previous section, the proposed method has been validated by simulation scenarios. In this section, the results of the experiments which were conducted for validation of the dynamic analysis of the flexible robot are presented. Figs. 15, 16 show the robot prototype and the system diagram of the robot used in the experiment. The two joints of the robot are actuated by a motor GB 37-3530 DC 12V (1) and a NEMA 17 step motor 200 steps/rev 12V (4), respectively. To collect the feedback signals, two rotary encoders LPD3806-600BM-G5-24V-2P-AB are used.

The experimental set-up also includes two flex sensors of 4.5-inch FSL0095-103ST. A Terminal Board STM32 F407/417 with the Cortex ${ }^{\text {TM}_{-}}$M4 core running at $168 \mathrm{MHz}$ was selected for the control hardware. The LabVIEW software interface was used to display the readings of the experiment. The two flex sensors $112.24 \mathrm{~mm}$ in length were adhered towards both ends of the flexible link. The maximum resistance of the sensors was $110 \mathrm{k} \Omega$. 

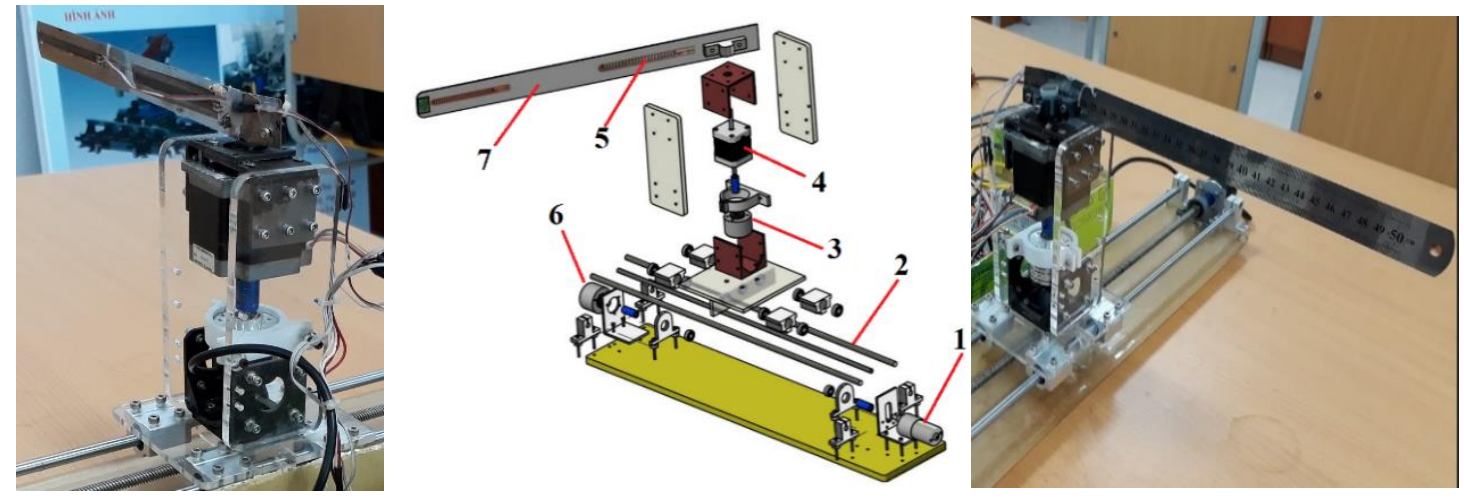

Figure 15. The flexible robot prototype for the experiment: (1) DC motor, (2) Lead screw, (3) Rotary encoder 1, (4) Step motor, (5) Flex sensor, (6) Rotary encoder 2, (7) Flexible link.

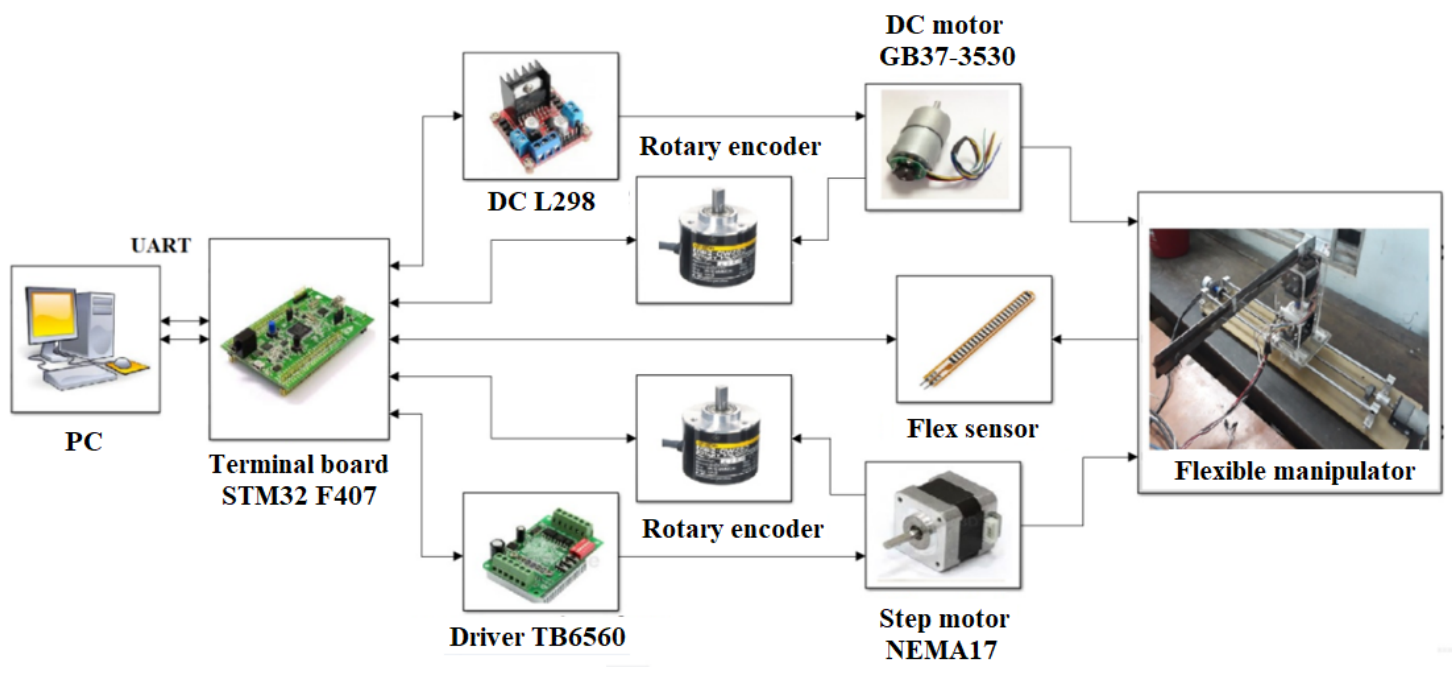

Figure 16. The systematic diagram of the robot for the experiment

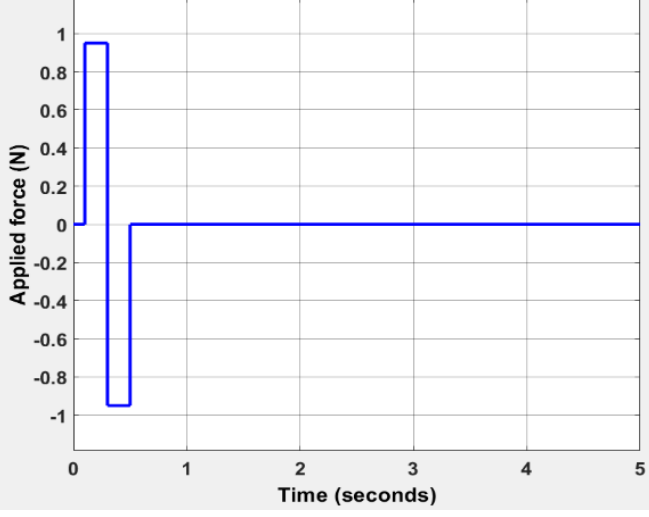

Figure 17. The applied force for the experiments

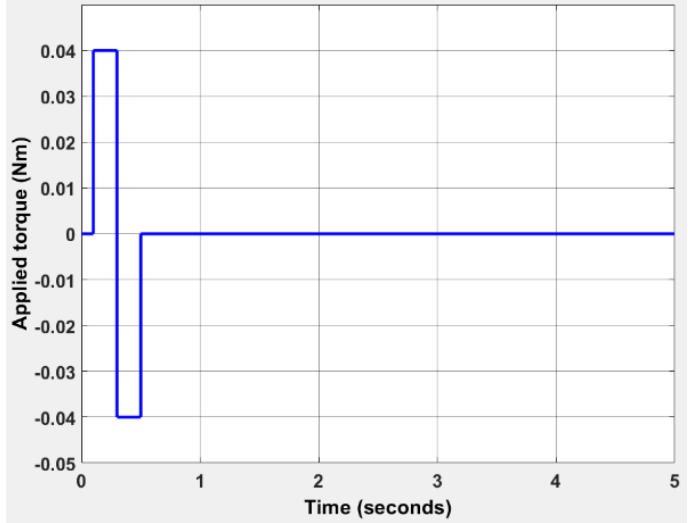

Figure 18. The applied torque for the experiments

Figs. 17 and 18 show the Bang-bang input signals (the applied force/torque) which were transformed into voltage and pulse signals for the motors. Fig. 19 and Fig 20 show the displacement of the joints produced by experiments and by simulation, respectively. It is observed that, in the steady-state, the deviation between the experimental curve and the computational curve is very small (about 3.5\% in magnitude). Fig. 21 presents the flexural displacement at the second node of the second element of link 2. Fig. 22 shows 
the flexural displacement at the arm tip. It can be seen that the shape and the value of the simulation curves, which were calculated by both the proposed method and the previous method closely matching the experimental readings. These results are a good validation of the dynamic modelling method proposed in this study.

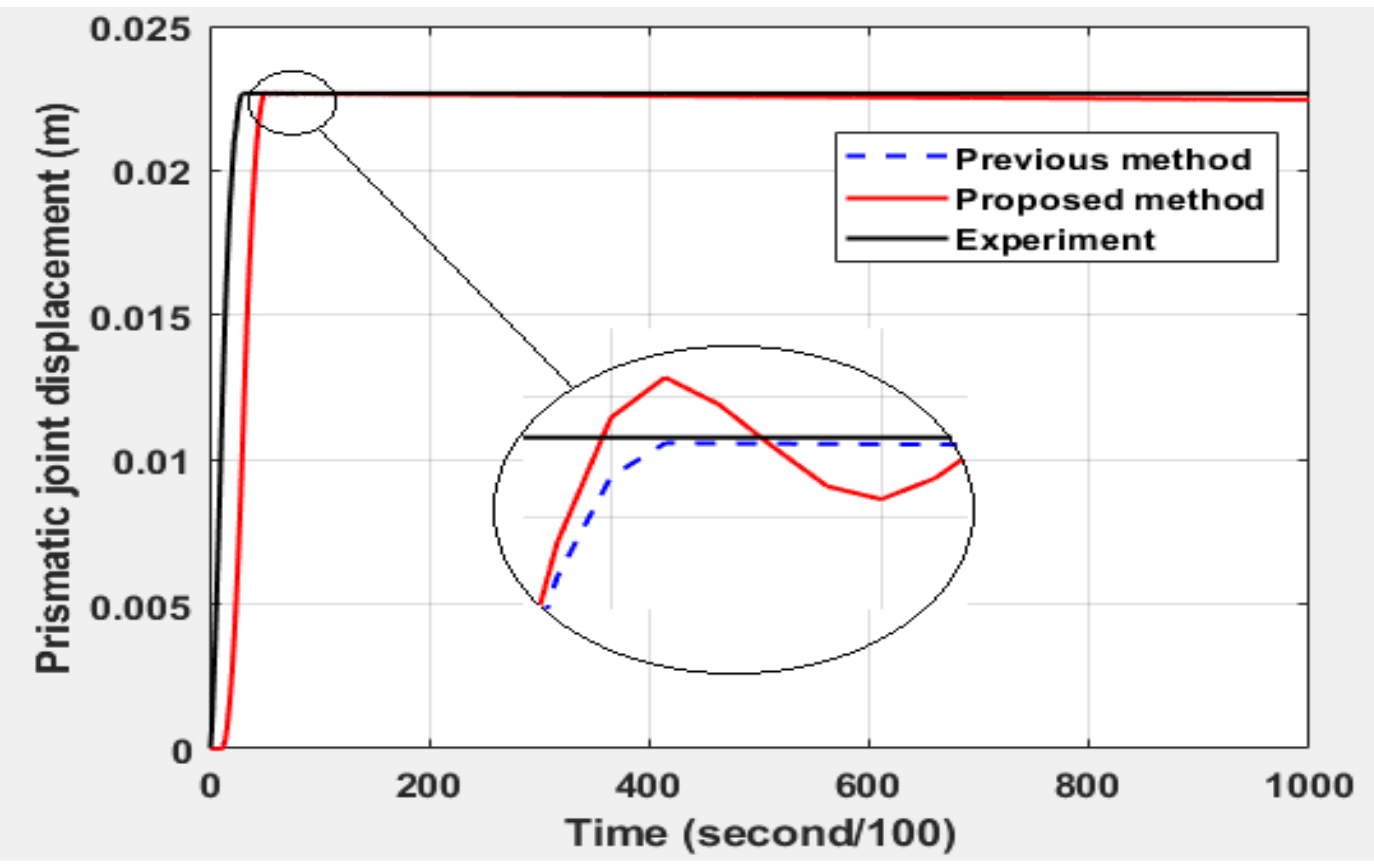

Figure 19. The prismatic joint displacement

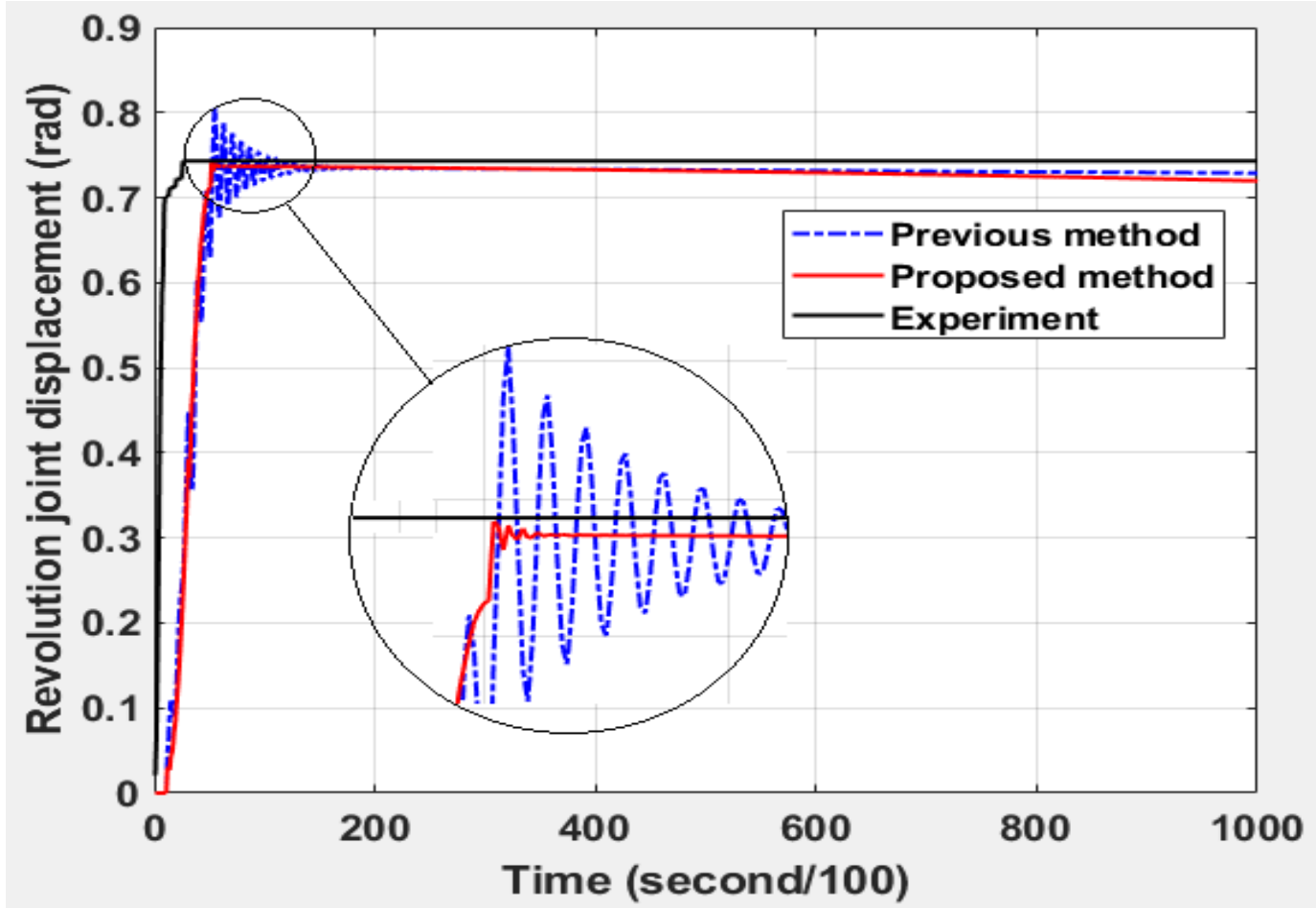

Figure 20. The revolute joint displacement 


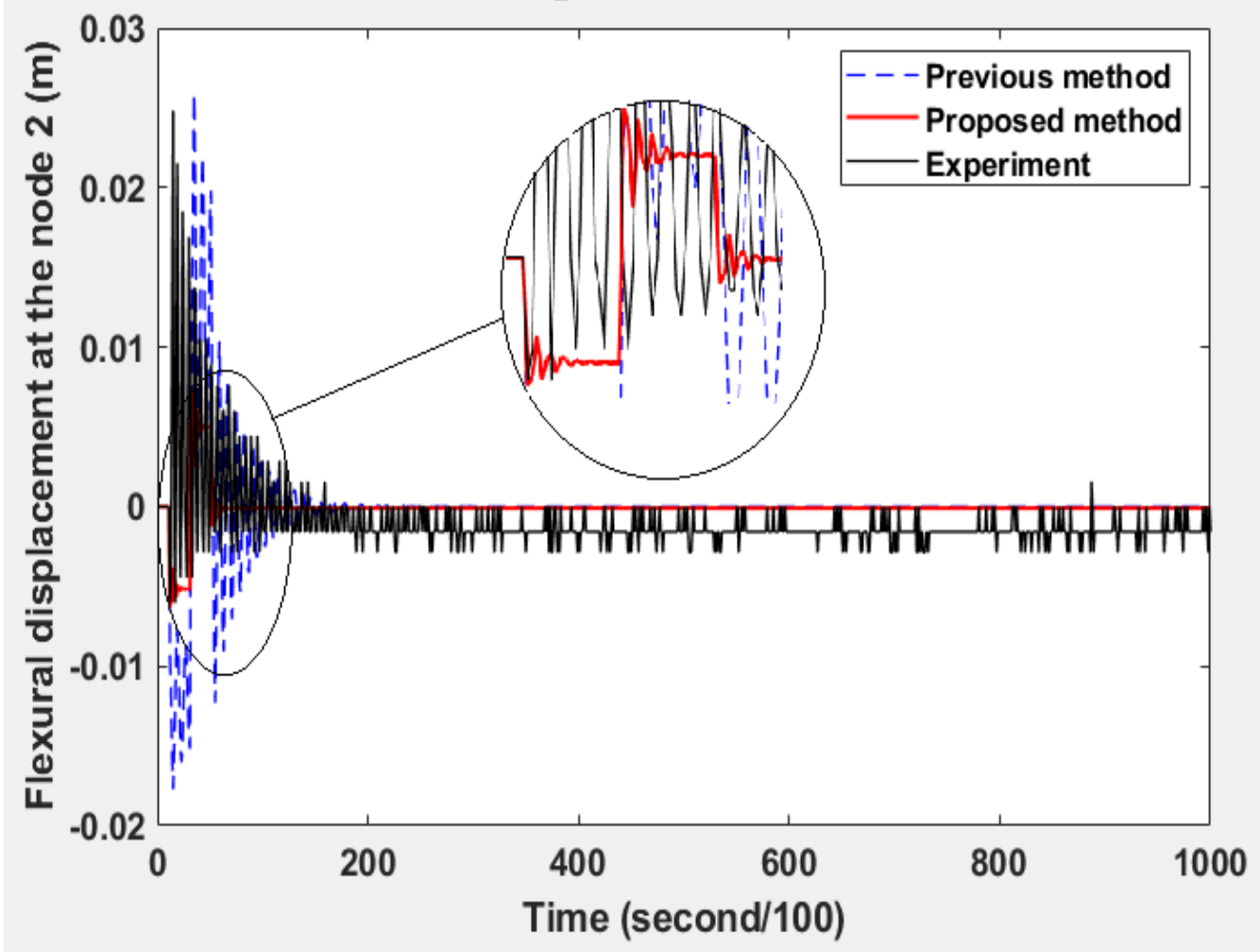

Figure 21. The flexural displacement at the second node of the second element of link 2

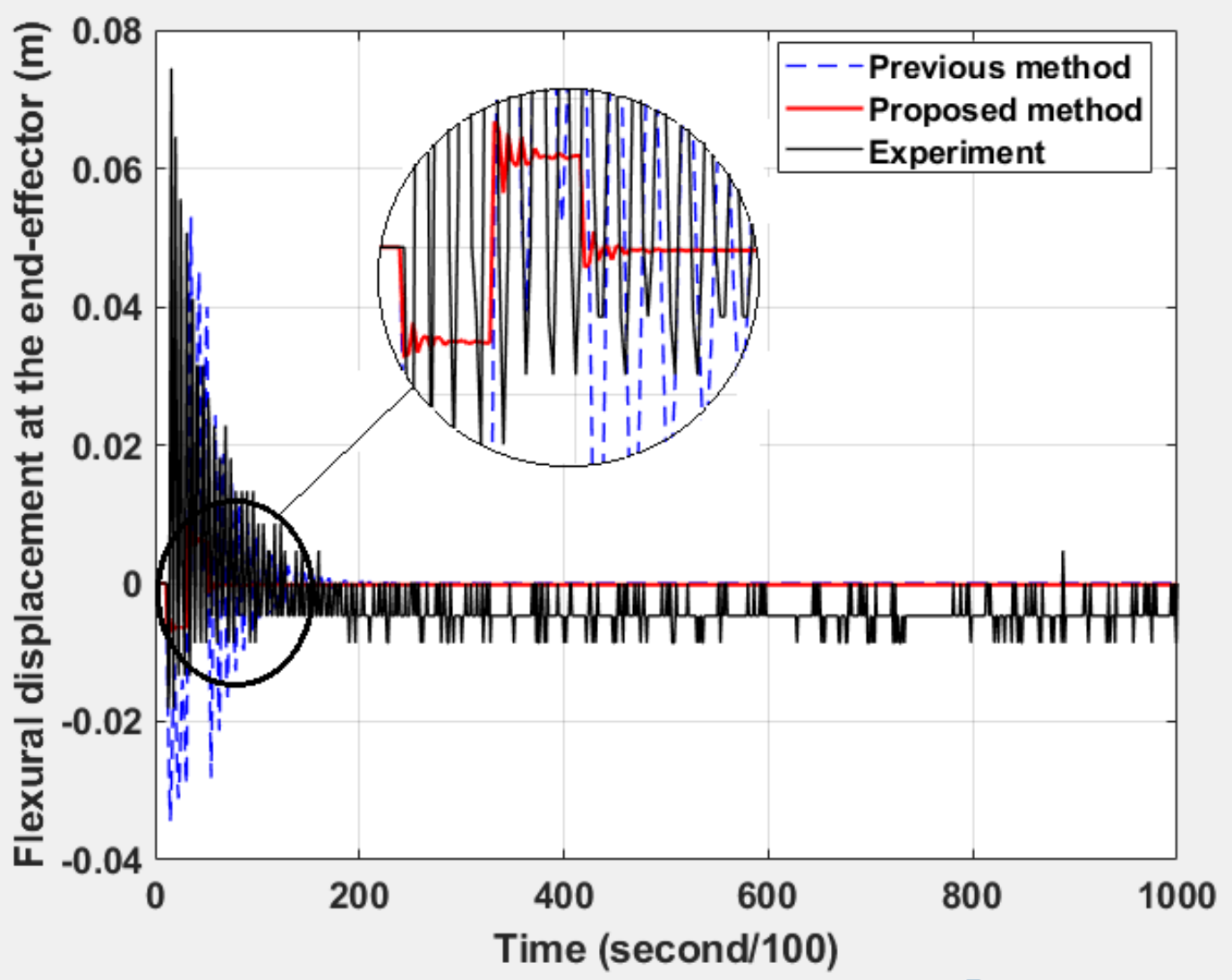

Figure 22. The flexural displacement at the end-effector 


\section{Discussion and Conclusion}

The simulation and experimental results have demonstrated the effectiveness and efficiency of the dynamic modelling method newly proposed in this study. In particular, as compared with the previous methods, the proposed method has two main advantages. The first advantage is that the dynamic equation of a flexible robot is derived in a simplified and explicit manner. In particular, the expression of the global mass matrices of the dynamic equation for a flexible robot and a rigid robot are similar. The second advantage of the method is that all the steps of the dynamic modelling procedure for a flexible robot is similar to the commonly used steps of the dynamic modelling procedure for the conventional rigid robot. Thus, the proposed method is very useful and helpful to users who have been familiar with the dynamic modelling and control of the rigid robots. A detailed comparison of the proposed method with the previously used methods is presented in Table 1.

Table 1. A comparison of the proposed method with the previously used methods

\begin{tabular}{|l|l|l|}
\hline \multicolumn{2}{|c|}{ FLEXIBLE ROBOTS } & \multicolumn{1}{c|}{ RIGID ROBOTS } \\
\hline $\begin{array}{l}\text { The previous methods [1, } \\
\mathbf{2 9 , 3 6 ]}\end{array}$ & The proposed method & $\begin{array}{c}\text { The conventional } \\
\text { methods }\end{array}$ \\
\hline $\mathbf{M}_{i j}=\int_{V_{i j}} m_{i}\left[\frac{\partial \mathbf{r}_{0 i j}}{\partial \mathbf{q}}\right]^{T}\left[\frac{\partial \mathbf{r}_{0 i j}}{\partial \mathbf{q}}\right] d V_{i j}$ & $\mathbf{M}_{i j}=m_{i} l_{i e} \mathbf{J}_{i j(T)}^{T} \mathbf{J}_{i j(T)}+\mathbf{J}_{i j(R)}^{T} \mathbf{I}_{i j} \mathbf{J}_{i j(R)}$ & $\begin{array}{l}\mathbf{M}_{i}=m_{l i} \mathbf{J}_{i(T)}^{T} \mathbf{J}_{i(T)}+\mathbf{J}_{i(R)}^{T} \mathbf{I}_{i} \mathbf{J}_{i(R)} \\
m_{l i} \text { and } \mathbf{I}_{i} \text { are the mass and } \\
\text { the matrix of the moment of } \\
\text { inertia of link } i\end{array}$ \\
\hline $\begin{array}{l}\text { Complex and implicit } \\
\text { transformations are needed } \\
\text { to formulate } \mathbf{M}_{i j} \text { and } \\
\text { construct } \mathbf{M} .\end{array}$ & $\mathbf{M}=\sum_{i=1}^{n} \sum_{j=1}^{n_{i}} \mathbf{M}_{i j}$ & $\mathbf{M}=\sum_{i=1}^{n} \mathbf{M}_{i}$ \\
\hline
\end{tabular}

It can be seen from Table 1 that, in the previous methods [1, 29, 36], each elemental mass matrix is usually computed and implicitly expressed in terms of nodal elastic deformations of a link because the symbolic integral of the element kinetic energy is employed. Not all the moment of inertia of an element and a link are included in the formulation. Moreover, the construction of the global mass matrix requires complex and implicit computational transformations. Whereas, in the proposed method, the mass and all the moment of inertia of all elements are taken into account. In addition, because the symbolic formulation of the dynamics model is simplified the calculation of the symbolic integrals and the implicit expressions of the elemental global mass matrices are not required. Furthermore, the global mass matrices for a flexible robot and a rigid robot are similar, which can be expressed in the same compact form ready for symbolic programming. Besides, the proposed method is even more efficient for the case when the number of links of a flexible robot increases and a large number of finite elements on the links is considered.

It is noticeable that, in our previous work [1], we proved a generic transformation matrix which characterizes the kinematics of a serial flexible robot constructed with three types of joints: the revolute joint, the fixed prismatic joint and the sliding prismatic joint. In addition, we addressed a new and effective algorithm of symbolic manipulation for 
constructing recursively the global mass and stiffness matrices of the dynamic equation for the flexible robots. In this proposed paper, we continue developing a mathematical scheme to formulate more effectively the dynamic equation of a flexible robot without implicit mathematical transformations and expressions. As a result, the methodology proposed in this study has some advantages over the previous methods, as discussed earlier.

In conclusion, a new mathematical approach for the dynamic modelling of flexible robots and flexible/rigid robots has been presented. The dynamic modelling method proposed in this study has been validated through numerical simulations and experimental results. A close match between the simulation and the experimental result has been shown clearly. The proposed method is more efficient and advantageous in comparison to the previous ones. It has also been shown that the proposed method is more useful when dealing with not only the flexible robots but also the hybrid flexible/rigid robots which consist of rigid links and flexible links simultaneously.

The application of the proposed methodology in order to analyse the dynamics and design control laws for more complex flexible robots will be the future works of this investigation.

\section{Acknowledgement:}

This work was supported by the Vingroup Innovation Foundation (VINIF) annual research support program under Grant VINIF. 2019.DA08.

\section{References}

1. My CA, Bien DX, Le CH, Packianather M. An efficient finite element formulation of dynamics for a flexible robot with different type of joints. Mechanism and Machine Theory. 2019;134: 267-88.

2. Sayahkarajy M, Mohamed Z, Mohd Faudzi AA. Review of modelling and control of flexible-link manipulators. Proc. Inst. Mech. Eng. Part I-J Syst Control Eng. 2016; 230(8): 861-873.

3. Jonker B. A finite element dynamic analysis of flexible manipulators. Int J Rob Res. 1990; 9(4): 59-74.

4. Du H, Lim MK, Liew KM. A nonlinear finite element model for dynamics of flexible manipulators. Mech Mach Theory. 1996; 31(8): 1109-1119.

5. Płosa J, Wojciech S. Dynamics of systems with changing configuration and with flexible beam-like links. Mech Mach Theory. 2000; 35(11): 1515-1534.

6. Naganathan G, Soni AH. Coupling effects of kinematics and flexibility in manipulators. Int J Rob Res. 1987; 6(1): 75-84.

7. Tokhi MO, Mohamed Z, Shaheed MH. Dynamic characterisation of a flexible manipulator system. Robotica. 2001; 19(5): 571-580.

8. Al-Bedoor BO, Almusallam AA. Dynamics of flexible-link and flexible-joint manipulator carrying a payload with rotary inertia. Mech Mach Theory. 2000; 35(6): 785-820.

9. Mahto S. Shape optimization of revolute-jointed single link flexible manipulator for vibration suppression. Mech Mach Theory. 2014; 75: 150-160.

10. Karagulle H, Malgaca L, Dirilmiş M, et al. Vibration control of a two-link flexible manipulator. Int J Rob Res. 2015; 23(12): 2023-2034. 
11. Karagulle H, Malgaca L, Dirilmiş M, et al. Vibration control of a two-link flexible manipulator. J Vib Control. 2017; 23(12): 2023-2034.

12. Al-Bedoor BO, Khulief YA. General planar dynamics of a sliding flexible link. J Sound Vib. 1997; 206(5): 641-661.

13. Pan YC, Scott RA, Ulsoy AG. Dynamic modeling and simulation of flexible robots with prismatic joints. J Mech Des. 1990; 112(3): 307-314.

14. Wang X, Mills JK. Dynamic modeling of a flexible-link planar parallel platform using a substructuring approach. Mech Mach Theory. 2006; 41(6): 671-687.

15. Usoro PB, Nadira R, Mahil SS. A finite element/Lagrange approach to modeling lightweight flexible manipulators. J Dyn Syst Meas Control. 1986; 108(3): 198-205.

16. Amirouche FML, Xie M. An explicit matrix formulation of the dynamical equations for flexible multibody systems: a recursive approach. Comput Struct. 1993; 46(2): 311-321.

17. Augustynek K, Adamiec-Wójcik I. Analysis of mechanisms with flexible beam-like links, rotary joints and assembly errors. Arch Appl Mech. 2012; 82(2): 283-295.

18. Pan, W., Mao, S., Haug, E. J., \& Solis, D. Efficient modal approach for flexible multibody dynamic simulation. Mechanics Based Design of Structures and Machines. 2003; 31(1): 1-23.

19. Zakhariev, E. Nonlinear dynamics of rigid and flexible multibody systems. Mechanics of Structures and Machines. 2000; 28(1): 105-136.

20. Theodore RJ, Ghosal A. Comparison of the AMM and FEM for flexible multi-link manipulators. Int J Rob Res. 1995; 14: 91-111.

21. Dwivedy SK, Eberhard P. Dynamic analysis of flexible manipulators, a literature review. Mech Mach Theory. 2006; 41(7): 749-777.

22. Hewit JR, Morris JR, Sato K and Ackermann F. Active force control of a flexible manipulator by distal feedback. Mechanism and machine theory. 1997; 32(5): 583596.

23. Mehrez MW and El-Badawy AA (2010) Effect of the joint inertia on selection of under-actuated control algorithm for flexible-link manipulators. Mechanism and Machine Theory. 2010; 45(7): 967-980.

24. Choi SB, Lee HB and Thompson BS. Compliant control of a two-link flexible manipulator by constraint Hamiltonian system. Mechanism and Machine Theory. 1998; 33(3): 293-306.

25. Subudhi B and Morris AS. Dynamic modelling, simulation and control of a manipulator with flexible links and joints. Robotics and Autonomous Systems. 2002; 41(4): 257-270.

26. Raouf F, Mohamad S, Maarouf S and Maamar B. Distributed adaptive control strategy for flexible link manipulators. Robotica. 2017; 35(7): 1562-1584.

27. Hermle, M., \& Eberhard, P. Control and parameter optimization of flexible robots. Mechanics of Structures and Machines. 2000; 28(2-3): 137-168.

27. Hong, S. M., \& Park, Y. S. Vibration Reduction of Flexible Manipulators Using Torque Wheels. Journal of Structural Mechanics. 1999; 27(1): 1-22.

28. Shabana AA. Flexible multibody dynamics: review of past and recent developments. Multibody System Dynamics. 1997;1(2):189-222.

29. Shabana A. Dynamics of multibody systems. Cambridge university press; 2020 Jan 31.

30. Shabana AA, Schwertassek R. Equivalence of the floating frame of reference approach and finite element formulations. International journal of non-linear mechanics. 1998 ;33(3):417-32. 
31. Garcia-Vallejo D, Sugiyama H, Shabana AA. Finite element analysis of the geometric stiffening effect. Part 1: a correction in the floating frame of reference formulation. Proceedings of the Institution of Mechanical Engineers, Part K: Journal of Multibody Dynamics. 2005;219(2):187-202.

32. Nada AA, Hussein BA, Megahed SM, Shabana AA. Use of the floating frame of reference formulation in large deformation analysis: experimental and numerical validation. Proceedings of the Institution of Mechanical Engineers, Part K: Journal of Multi-body Dynamics. 2010;224(1):45-58.

33. Shabana AA, Yakoub RY. Three dimensional absolute nodal coordinate formulation for beam elements: theory. J. Mech. Des.. 2001 Dec 1;123(4):606-13.

34. Berzeri M, Campanelli M, Shabana AA. Definition of the elastic forces in the finiteelement absolute nodal coordinate formulation and the floating frame of reference formulation. Multibody System Dynamics. 2001;5(1):21-54.

35. Gerstmayr J. Comparison of the absolute nodal coordinate and the floating frame of reference formulation by means of a simplified strain formulation. In ASME 2003 International Design Engineering Technical Conferences and Computers and Information in Engineering Conference 2003 Jan 1 (pp. 29-35). American Society of Mechanical Engineers Digital Collection.

36. Kermanian A, Kamali A, Taghvaeipour A. Dynamic analysis of flexible parallel robots via enhanced co-rotational and rigid finite element formulations. Mechanism and Machine Theory. 2019;139:144-73.

37. Elkaranshawy HA, Dokainish MA. Corotational finite element analysis of planar flexible multibody systems. Computers \& structures. 1995; 54.5: 881-890.

38. Hsiao K. M., Lin J. Y., \& Lin W. Y. A consistent co-rotational finite element formulation for geometrically nonlinear dynamic analysis of 3-D beams. Computer Methods in Applied Mechanics and Engineering. 1999; 169.1-2: 1-18.

39. Urthaler Y., \& Reddy J. N. A corotational finite element formulation for the analysis of planar beams. Communications in numerical methods in engineering. 2005; 21.10: 553-570.

40. Felippa C. A., \& Haugen B. A unified formulation of small-strain corotational finite elements: I. Theory. Computer Methods in Applied Mechanics and Engineering. 2005; 194.21-24: 2285-2335.

41. Tsai M. H., Lin W. Y., Zhou Y. C., \& Hsiao K. M. A corotational finite element method combined with floating frame method for large steady-state deformation and free vibration analysis of a rotating-inclined beam. Mathematical Problems in Engineering. 2011; 2011:1-29.

42. Le T. N., Battini J. M., \& Hjiaj M. A consistent 3D corotational beam element for nonlinear dynamic analysis of flexible structures. Computer Methods in Applied Mechanics and Engineering. 2014; 269: 538-565.

43. Wittbrodt E., Adamiec-Wójcik I., \& Wojciech S. Dynamics of flexible multibody systems: rigid finite element method. Springer Science \& Business Media. 2007.

44. Shabana A.A. Finite element incremental approach and exact rigid body inertia. ASME Journal of Mechanical Design. 1996; 118.2: 171-178.

45. Shabana A.A. An absolute nodal coordinate formulation for the large rotation and deformation analysis of flexible bodies. Technical Report No. MBS96-1-UIC, Department of Mechanical Engineering, University of Illinois at Chicago. 1996.

46. Shabana A.A. Vibration of Discrete and Continuous Systems, 2nd edn. SpringerVerlag, New York, 1997. 